\title{
A FORMAL EXPANSION PROCEDURE FOR THE SOLITARY WAVE PROBLEM*
}

\author{
By BERNARD EPSTEIN (University of New Mexico)
}

1. The (two-dimensional) problem of the solitary wave may be stated as follows: to demonstrate the existence and to determine the shape of a profile which moves over a horizontal line without change of shape at a constant speed, the fluid being assumed incompressible and inviscid. A detailed formulation of the problem, together with an existence proof, has been presented by Friedrichs and Hyers [1]. Their remarkable paper also presents a brief survey of earlier work on the problem, together with an extensive bibliography of the pertinent literature.

Let the horizontal line over which the flow occurs be taken as the $x$-axis and let the asymptotic height of the profile and the velocity at infinity be denoted by $h$ and $U$ respectively. Then by change of scale these two quantities may be normalized to unity, and it becomes apparent that only a single essential parameter appears in the problem, namely $g h / U^{2}$, where $g$ denotes the gravitational acceleration; this parameter is henceforth denoted by $\gamma$.

The mathematical problem may now be formulated as follows. A nontrivial function $f(x)$ which vanishes at infinity is sought such that the following system of equations for the unknown function $\psi(x, y)$, the streamfunction of the flow, is solvable:

$$
\begin{gathered}
\Delta \psi=0 \text { in the region } 0<y<1+f(x) ; \\
\partial \psi / \partial x \rightarrow 0 \text { and } \partial \psi / \partial y \rightarrow 1 \text { as }|x| \rightarrow \infty ; \\
\psi(x, 0) \equiv 0, \quad \psi(x, 1+f(x))=1 ;
\end{gathered}
$$

on the free streamline, defined by the equation $y=1+f(x)$, the Bernoulli condition $\partial \psi / \partial n=(1-2 \gamma f(x))^{1 / 2}$ must be satisfied, where $\partial / \partial n$ denotes differentiation in the direction of the outward normal.

In numerous treatments of this problem antedating [1], nonrigorous but physically plausible arguments have indicated that a symmetric solution of this problem does exist for values of $\gamma$ less than but sufficiently close to unity - that is, for values of $U$ slightly exceeding the "critical velocity" $(g h)^{1 / 2}$-and that the function $f(x)$ defining the shape of the profile is given, in some approximate sense, by

$$
f(x) \simeq \frac{4}{3} a^{2} \operatorname{sech}^{2} a x
$$

* Received November 5, 1972. Research partially supported by the National Science Foundation under Grant GP 29042. The author wishes to thank Dr. I. I. Kolodner, who suggested the idea of extending the definition of the stream-function to the entire plane and then employing a simple-layer representation of the entire function. A portion of this work was done during the academic year 197172 when the author was on sabbatical leave at Technion (Israel Institute of Technology). 
where $\gamma=\exp \left(-\frac{4}{3} a^{2}\right)=\sum_{n=0}^{\infty}\left(-\frac{4}{3} a^{2}\right)^{n} / n$ !. (In [1] the authors define $\gamma$ by the equation $\gamma=\exp \left(-3 a^{2}\right)$, so that the "principal term" has a slightly different form in their treatment, namely $3 a^{2} \operatorname{sech}^{2} \frac{3}{2} a x$.)

In the following section the problem formulated above in Eqs. (1a-d) is converted into a nonlinear integrodifferential equation, which is then analyzed at some length in subsequent sections. In particular, the approximation (2) is derived, and a procedure is developed for deriving a formal solution in the form of an infinite series. Indeed, it is shown that by altering slightly the relationship between $\gamma$ and $a$ which is given above immediately after (2), we can assure that the higher-order terms of the series assume an especially simple form. We have not succeeded in demonstrating that this series is actually convergent (for sufficiently small values of $a$ ), but the comparative simplicity of the terms of the expansion gives some cause to hope that a more precise investigation will indeed establish the convergence. Of course, it may very well happen that the series expansion, while divergent for all non-zero values of $a$, is asymptotically correct.

2. Assuming that the problem defined by Eqs. (1a-d) is solvable, we extend the definition of $\psi(x, y)$ to the entire plane as follows: $\psi(x, y) \equiv 1$ when $y>1+f(x)$, $\psi(x, y) \equiv-\psi(x,-y)$ when $y<0$. By taking account of (1a), (1c), and the Schwarz reflection principle, one sees that $\psi(x, y)$ is continuous everywhere, and that it is harmonic everywhere except on the profile and on its image $(y=-1-f(x))$. On these two curves the normal derivative undergoes a jump-discontinuity of $(1-2 \gamma f(x))^{1 / 2}$, so that a simple layer of density $\pm(1 / 2 \pi)(1-2 \gamma f(x))^{1 / 2}$ is associated with $\psi(x, y)$ along these curves, the signs being chosen positive and negative along the profile and its image respectively. (Since $\psi(x, y)$, unlike its gradient, experiences no discontinuity, no double layer is involved.) Taking account of the behavior of $\psi(x, y)$ "at infinity" and of the oddness (with respect to $y$ ) of this function, one can easily justify the assertion that $\psi(x, y)$ is actually equal to the potential generated by the aforementioned mass-distribution, and so we obtain the integral representation

$\psi(x, y)=\frac{1}{2 \pi} \int_{-\infty}^{\infty}(1-2 \gamma f(\xi))^{1 / 2}\left(1+f^{\prime}(\xi)^{2}\right)^{1 / 2} \log \left\{\frac{(\xi-x)^{2}+(y+1+f(\xi))^{2}}{(\xi-x)^{2}+(y-1)-f(\xi)^{2}}\right\}^{1 / 2} d \xi$.

In particular, for $y>1+f(x)$, and $a$ fortiori for $y>1+\max f(x)$, the identity

$$
4 \pi \stackrel{x, \nu}{\equiv} \int_{-\infty}^{\infty}(1-2 \gamma f(\xi))^{1 / 2}\left(1+f^{\prime}(\xi)^{2}\right)^{1 / 2} \log \left(\frac{(\xi-x)^{2}+(y+1+f(\xi))^{2}}{(\xi-x)^{2}+(y-1-f(\xi))^{2}}\right\} d \xi
$$

must hold. (In particular, if the trivial solution $f(x) \equiv 0$ is inserted into (3), one obtains for $\psi(x, y)$ the values $1, y$, and -1 under the conditions $y>1,|y|<1$, and $y<-1$, respectively, in agreement with (1a-d) and (4).) Conversely, if the function $f(x)$ satisfies the integrodifferential equation (4) and behaves suitably at infinity, then it is not difficult to see that the function $\psi(x, y)$ defined by (3) constitutes (for $0<y<1+f(x)$ ) a solution of the system (1a-d).

3. If for convenience we momentarily set

$$
u=\frac{i(1+f(\xi))}{\xi-x+i y}
$$

then the logarithm appearing in (4) can be expressed as $\log |1+u / 1-u|^{2}$, or 
$2 \operatorname{Re} \log (1+u) /(1-u)$. The restriction imposed on $y$, namely that $y>1+\max f(x)$, guarantees that $|u|<1$, so that

$$
2 \log \frac{1+u}{1-u}=4\left(u+\frac{u^{3}}{3}+\frac{u^{5}}{5}+\cdots\right) .
$$

Substituting from (5) into (6) and taking the real part of the resulting series, we obtain, by an elementary computation,

$$
\log \frac{(\xi-x)^{2}+(y+1+f(\xi))^{2}}{(\xi-x)^{2}+(y-1-f(\xi))^{2}}=4 \pi \sum_{n=0}^{\infty} \frac{(-1)^{n}(1+f(\xi))^{2 n+1}}{(2 n+1) !} \frac{\partial^{2 n} P(\xi-x, y)}{\partial \xi^{2 n}},
$$

where $P(\xi-x, y)$ is the Poisson kernel of the upper half-plane, namely $y / \pi\left[(\xi-x)^{2}+y^{2}\right]$. Substituting from (7) into (4) we obtain, for $y>1+\max f(x)$, the identity

$$
1 \stackrel{x, y}{\equiv} \int_{-\infty}^{\infty}(1-2 f(\xi))^{1 / 2}\left(1+f^{\prime}(\xi)^{2}\right)^{1 / 2} \sum_{n=0}^{\infty} \frac{(-1)^{n}(1+f(\xi))^{2 n+1}}{(2 n+1) !} \frac{\partial^{2 n} P(\xi-x, y)}{\partial \xi^{2 n}} d \xi
$$

If it is assumed that the function $f(\xi)$ possesses derivatives of all orders and that these derivatives vanish at infinity, then the same is true for all derivatives of

$$
(1-2 \gamma f(\xi))^{1 / 2}\left(1+f^{\prime}(\xi)^{2}\right)^{1 / 2}(1+f(\xi))^{2 n+1}
$$

Moving the summation outside the integration, integrating by parts so as to transfer all the differentiations from the Poisson kernel to the remaining factor in each term of the summation, taking account of the conditions at infinity, and then restoring the order of integration and summation, we obtain (entirely formally)

$$
1 \stackrel{x, y}{\equiv} \int_{-\infty}^{\infty} g(\xi) P(\xi-x, y) d \xi
$$

where

$$
g(\xi)=\sum_{n=0}^{\infty} \frac{(-1)^{n}}{(2 n+1) !} \frac{d^{2 n}}{d \xi^{2 n}}\left\{(1-2 \gamma f(\xi))^{1 / 2}\left(1+f^{\prime}(\xi)^{2}\right)^{1 / 2}(1+f(\xi))^{2 n+1}\right\} .
$$

Assuming that (10) does indeed define a bounded and continuous function $g(\xi)$, we argue as follows. The right-hand side of (9) exists for all points in the upper half-plane and defines there a harmonic function. According to (9), this harmonic function must be identically equal to unity in the half-plane $y>1+\max f(x)$; now, by the uniqueness theorem for harmonic functions, this harmonic function must equal unity everywhere in the upper half-plane. Thus the boundary values of this function are identically equal to unity, while by (9) they are given by $g(\xi)$. Hence, we conclude, quite formally, that the function which defines the shape of the profile must satisfy the following differential equation (of infinite order!):

$$
\sum_{n=0}^{\infty} \frac{(-1)^{n}}{(2 n+1) !} \frac{d^{2 n}}{d \xi^{2 n}}\left\{(1-2 \gamma f(\xi))^{1 / 2}\left(1+f^{\prime}(\xi)^{2}\right)^{1 / 2}(1+f(\xi))^{2 n+1}\right\}=1
$$

4. We now indicate how it is possible to derive from (11) the "principal term" given by (2)-in fact, a formal expansion for the shape of the profile. It appears that a "stretching technique" is quite indispensable in connection with this problem (cf. [1]). We introduce the new variables $\eta=a \xi$ and $F(\eta)=f(\xi)$, where $a$ is defined in Sec. 1 . 
Then (11) assumes the form

$$
\sum_{n=0}^{\infty} \frac{(-1)^{n} a^{2 n}}{(2 n+1) !} \frac{d^{2 n}}{d \eta^{2 n}}\left\{(1-2 \gamma F(\eta))^{1 / 2}\left(1+a^{2} F^{\prime}(\eta)^{2}\right)^{1 / 2}(1+F(\eta))^{2 n+1}\right\}=1 .
$$

We assume for $F(\eta)$ an expansion of the form

$$
F(\eta)=a \phi_{1}(\eta)+a^{2} \phi_{2}(\eta)+a^{3} \phi_{3}(\eta)+\cdots,
$$

insert this expansion into (12), and assemble all terms containing a specified power of $a$. (The presence of the factor $a^{2 n}$ assures that each power of $a$ will be multiplied by a polynomial expression in the $\phi_{k}$ 's and their derivatives.) The coefficients of $a^{0}, a^{1}$, and $a^{2}$ turn out to be 1,0 , and $-\frac{3}{2} \phi_{1}{ }^{2}$ respectively. Thus, matching of coefficients on the two sides of (12) leads to the conclusion that $\phi_{1}(\eta)$ must vanish identically. The exact form of the coefficient of $a^{3}$ need not concern us here; what does matter is that it vanishes identically when account is taken of the fact that $\phi_{1}$ must vanish identically. Next, the coefficient of $a^{4}$ is found to equal $\frac{4}{3} \phi_{2}-\frac{3}{2} \phi_{2}{ }^{2}-\frac{1}{3} \phi_{2}{ }^{\prime \prime}$ (plus terms which vanish identically by virtue of the vanishing of $\left.\phi_{1}\right)$. Hence, we are led to the differential equation

$$
\frac{4}{3} \phi_{2}-\frac{3}{2} \phi_{2}^{2}-\frac{1}{3} \phi_{2}^{\prime \prime}=0 .
$$

A simple analysis shows that, aside from the trivial solution, Eq. (14) possesses the following one-parameter family of solutions which vanish at $\pm \infty$ (and no others):

$$
\phi_{2}(\eta)=\frac{4}{3} \operatorname{sech}^{2}(\eta-\text { constant }) \text {. }
$$

Since the arbitrary constant appearing in (15) can be eliminated by a translation, we may consider that (14) possesses the unique non-trivial "well-behaved" solution

$$
\phi_{2}(\eta)=\frac{4}{3} \operatorname{sech}^{2} \eta
$$

in agreement with (2).

5. The coefficient of $a^{5}$ works out to $\frac{4}{3} \phi_{3}-3 \phi_{2} \phi_{3}-\frac{1}{3} \phi_{3}{ }^{\prime \prime}$, when one takes account of the identical vanishing of $\phi_{1}$. The differential equation

$$
\frac{4}{3} \phi_{3}-3 \phi_{2} \phi_{3}-\frac{1}{3} \phi_{3}{ }^{\prime \prime}=0
$$

possesses the "well-behaved" nontrivial solution $\operatorname{sech}^{2} \eta \tanh \eta$ (when $\phi_{2}$ is replaced by the right-hand side of $\left(15^{\prime}\right)$ ). It readily follows from this that there does not exist a nontrivial even solution of (16) which vanishes at infinity, for otherwise all solutions of (16) would vanish at infinity, and this is certainly not true. Since we are confining attention to the search for a symmetric profile, we are led to the conclusion that $\phi_{3}$ vanishes identically. However, we remark that the existence of a nontrivial odd solution of (16) which vanishes at infinity may indicate the existence of a non-symmetric solitary wave. Also, we remark that if for $\phi_{2}$ we had chosen the trivial function instead of the "principal term" we would still be led to the conclusion that $\phi_{3}$ must vanish identically (even without the restriction that $\phi_{3}$ should be an even function).

Before writing down the coefficient of $a^{6}$ we remark that all the results obtained up to this point remain unchanged if the relation between $\gamma$ and $a$ is given by any power series of the form

$$
\gamma=1-\frac{4}{3} a^{2}-c_{2} a^{4}-c_{3} a^{6}-\cdots
$$


rather than the specific one appearing after (2), for the values of $c_{2}, c_{3}, \cdots$ have not yet played any role. We temporarily employ the form (17) in order to determine whether some specific choice of the coefficients $c_{2}, c_{3}, \ldots$ will simplify the search for the coefficients $\phi_{4}, \phi_{5}, \cdots$ appearing in the expansion (13). The coefficient of $a^{6}$ now works out to (when one uses the identities $\phi_{1} \equiv \phi_{3} \equiv 0$ ):

$$
\begin{aligned}
\frac{4}{3} \phi_{4}-3 \phi_{2} \phi_{4} & -\frac{1}{3}{\phi_{4}}^{\prime \prime} \\
+ & \left\{c_{2} \phi_{2}+\frac{8}{3}{\phi_{2}}^{2}-{\phi_{2}}^{3}+\frac{1}{2}{\phi_{2}}^{\prime 2}+\frac{1}{30}{\phi_{2}}^{\prime \prime \prime \prime}-\frac{2}{9}{\phi_{2}}^{\prime \prime}+\frac{1}{12}\left(\phi_{2}^{2}\right)^{\prime \prime}\right\} .
\end{aligned}
$$

When one takes account of $\left(15^{\prime}\right)$ one sees that the expression in braces assumes the form $A \operatorname{sech}^{2} \eta+B \operatorname{sech}^{4} \eta+C \operatorname{sech}^{6} \eta$, where $A$, but neither $B$ nor $C$, depends on $c_{2}$. If $c_{2}$ is so chosen that $A$ vanishes, the differential equation obtained by equating (18) to zero possesses a unique even well-behaved solution, and this solution is of the form $\alpha \operatorname{sech}^{2} \eta+\beta \operatorname{sech}^{4} \eta$ (cf. sec. 6); for any other choice of $c_{2}$ there will still exist a unique even well-behaved solution, but it will not possess such a simple form. A simple calculation shows that the value of $c_{2}$ for which the aforementioned simple solution exists is given by $c_{2}=16 / 45$; with this choice of $c_{2}$, one obtains for $\phi_{4}$ the expression

$$
\phi_{4}=\frac{8}{9} \operatorname{sech}^{2} \eta+\frac{4}{3} \operatorname{sech}^{4} \eta \text {. }
$$

Proceeding still further, we obtain as the coefficient of $a^{7}$ the expression $\frac{4}{3} \phi_{5}-$ $3 \phi_{2} \phi_{5}-\frac{1}{3} \phi_{5}{ }^{\prime \prime}$, and the argument employed previously leads to the conclusion that $\phi_{5}$, like $\phi_{1}$ and $\phi_{3}$, must vanish identically. Similarly, all further $\phi_{k}$ 's with odd index are eliminated.

The coefficient of $a^{8}$ assumes the form (when one takes account of the specific forms of $\phi_{2}$ and $\phi_{4}$ )

$$
\frac{4}{3} \phi_{6}-3 \phi_{2} \phi_{6}-\frac{1}{3} \phi_{6}{ }^{\prime \prime}+\left\{A \operatorname{sech}^{2} \eta+B \operatorname{sech}^{4} \eta+C \operatorname{sech}^{6} \eta+D \operatorname{sech}^{8} \eta\right\},
$$

where $A$ involves the coefficient $c_{3}$ appearing in (17), while $B, C, D$ are completely fixed. Once again it is easily shown that for exactly one choice of $c_{3}$, namely $128 / 945$, the coefficient $A$ vanishes and the differential equation obtained by equating (20) to zero possesses a unique solution of the simple form $\alpha_{1} \operatorname{sech}^{2} \eta+\alpha_{2} \operatorname{sech}^{4} \eta+\alpha_{3} \operatorname{sech}^{6} \eta$, namely:

$$
\phi_{6}=\frac{8}{9} \operatorname{sech}^{2} \eta-\frac{4}{135} \operatorname{sech}^{4} \eta+\frac{404}{135} \operatorname{sech}^{6} \eta .
$$

The rest of the procedure is obvious. We disregard the coefficients of $a^{9}, a^{11}, a^{13}, \ldots$, but as we determine the coefficients of $a^{10}, a^{12}, a^{14}, \cdots$ we find that there exists a unique choice of the coefficients $c_{4}, c_{5}, c_{6}, \cdots$ for which the functions $\phi_{8}, \phi_{10}, \phi_{12}, \cdots$ turn out to be polynomials of degree $4,5,6, \ldots$ in $\operatorname{sech}^{2} \eta$. If this procedure is to have any meaning, we must show that when the coefficients of the series (17) are chosen in the manner described above, the series will have a positive radius of convergence, and it would be even better if one could determine explicitly all the coefficients at once, rather than by employing a tedious recurrence procedure. As shown in Sec. 7, the coefficients are given by

$$
c_{k}=\frac{2^{2 k+1} \zeta(2 k)}{\pi^{2 k}}, \quad \zeta(2 k)=\sum_{n=1}^{\infty} n^{-2 k}
$$


and the series (17) converges, for $|a|<\pi / 2$, to $2 a \cot 2 a$, so that $\gamma$ decreases monotonely from one to zero as $a$ increases from zero to $\pi / 4$.

6. It is evident, by induction, that if the functions $\phi_{2}, \phi_{4}, \cdots, \phi_{2 n-2}$ are given by polynomials of degree $1,2, \cdots, n-1$ in $\operatorname{sech}^{2} \eta$, then the coefficient of $a^{2 n+2}$ in the expansion of the left-hand side of (12) will assume the form

$$
\frac{4}{3} \phi_{2 n}-3 \phi_{2} \phi_{2 n}-\frac{1}{3} \phi_{2 n}{ }^{\prime \prime}+\sum_{k=1}^{r} A_{k, n} \operatorname{sech}^{2 k} \eta \text {. }
$$

If we insert for $\phi_{2 n}$ the expression $\sum_{k=1}{ }^{n} B_{k, n} \operatorname{sech}^{2 k} \eta$ (and, of course, for $\phi_{2}$ the expression $\frac{4}{3} \operatorname{sech}^{2} \eta$ ), then (23) assumes the form

$$
\begin{aligned}
A_{1, n} \operatorname{sech}^{2} \eta & +\left\{A_{n+1, n}+\frac{4 n^{2}+2 n-12}{3} B_{n, n}\right\} \operatorname{sech}^{2 n+2} \eta \\
& +\sum_{k=2}^{n}\left\{\frac{4}{3}\left(1-k^{2}\right) B_{k, n}+\frac{4 k^{2}-6 k-10}{3} B_{k-1, n}+A_{k, n}\right\} \operatorname{sech}^{2 k} \eta .
\end{aligned}
$$

From (24) it is immediately evident that the differential equation obtained by equating the expression (23) to zero possesses a solution which is a polynomial in $\operatorname{sech}^{2} \eta$ if and only if $A_{1, n}$ vanishes; when this condition is satisfied, the polynomial will be of degree $n$, the coefficient $B_{n, n}$ being given by $-3 A_{n+1, n} /\left(4 n^{2}+2 n-12\right)$ and the remaining coefficients then being obtained recursively from the equations

$$
B_{k-1, n}=\frac{3}{4 k^{2}-6 k-10}\left\{A_{k, n}+\frac{4}{3}\left(1-k^{2}\right) B_{k, n}\right\}, \quad 2<k<n .
$$

Indeed, by carefully examining (25) and the preceding formula for $B_{n, n}$ one can show that

$$
\left[\begin{array}{c}
B_{1, n} \\
B_{2, n} \\
\vdots \\
B_{n, n}
\end{array}\right]=3\left[\begin{array}{cccc}
c_{11} & c_{12} & \cdots & c_{1 n} \\
c_{21} & c_{22} & \cdots & c_{2 n} \\
\cdot & \cdot & \cdot & \cdot \\
\cdot & \cdot & \cdot & \cdot \\
c_{n 1} & c_{n 2} & \cdots & c_{n n}
\end{array}\right]\left[\begin{array}{c}
A_{2, n} \\
A_{3, n} \\
\vdots \\
\vdots \\
A_{n+1, n}
\end{array}\right],
$$

where the matrix-elements $c_{i j}$ are defined as follows: First, we define $a_{k}$ as $(k+2)(2-4 k)$ and $b_{k}$ as $4 k(k+2)$, and then we define $c_{i i}$ as zero if $i<j, c_{i i}$ as $1 / a_{i}, c_{i, i+1}$ as $-b_{i} / a_{i} a_{i+1}, c_{i, i+2}$ as $b_{i} b_{i+1} / a_{i} a_{i+1} a_{i+2}, c_{i, i+3}$ as $-b_{i} b_{i+1} b_{i+2} / a_{i} a_{i+1} a_{i+2} a_{i+3}$, etc. (Note that the index $n$ does not enter at all into these definitions.)

It is hoped that it may prove possible, by exploiting the rather simple form of the matrix appearing in (26), to obtain by an inductive argument bounds on the coefficients $A_{i, n}(2<i<n+1)$ and $B_{i, n}(1<j<n)$ which, in turn, will guarantee the convergence of the formal expansion (13) for sufficiently small values of the parameter $a$.

7. Finally, we proceed to justify the assertion made in Sec. 5 concerning the "optimal" choice of the coefficients appearing in (17). It is not difficult to see that the problem of choosing the $c_{k}$ 's is unaffected by linearizing Eq. (12). That is, we omit the radical $\left(1+a^{2} F^{\prime}(\eta)^{2}\right)^{1 / 2}$, replace $(1-2 \gamma F(\eta))^{1 / 2}$ and $(1+F(\eta))^{2 n+1}$ by $1-\gamma F(\eta)$ and $1+$ 
$(2 n+1) F(\eta)$, respectively, and finally omit the quadratic term appearing in the product of the latter two expressions. Thus, for the linearized version of (12) we obtain

$$
\sum_{n=0}^{\infty} \frac{(-1)^{n} a^{2 n}}{(2 n+1) !} \frac{d^{2 n}}{d \eta^{2 n}}\{1+(2 n+1-\gamma) F(\eta)\} \equiv 1 .
$$

Since each of the functions $\phi_{1}, \phi_{2}, \phi_{3} \cdots$ appearing in the expansion (13) is to be a polynomial in $\operatorname{sech}^{2} \eta, F(\eta)$ must, for large (positive) $\eta$, be expressible (at least formally) as a series of the form

$$
F(\eta)=\alpha_{1} \exp (-2 \eta)+\alpha_{2} \exp (-4 \eta)+\cdots,
$$

where the coefficients $\alpha_{1}, \alpha_{2}, \cdots$ will, of course, depend on the parameter $a$. Inserting this expansion into $\left(12^{\prime}\right)$, one finds that the left-hand side of the latter reduces to

$$
1+\alpha_{1} \exp (-2 \eta) \sum_{n=0}^{\infty} \frac{(-1)^{n}(2 a)^{2 n}(2 n+1-\gamma)}{(2 n+1) !}+\cdots,
$$

where the terms indicated by $\cdots$ contain powers (greater than the first) of $\exp (-2 \eta)$, and hence are negligible in comparison with exp $(-2 \eta)$. Thus, we obtain (since $\alpha_{1}$ certainly does not vanish)

$$
\sum_{n=0}^{\infty} \frac{(-1)^{n}(2 a)^{2 n}(2 n+1-\gamma)}{(2 n+1) !}=0,
$$

and this equation is exactly equivalent to $\gamma=2 a \cot 2 a$, in agreement with (22).

\section{REFERENCE}

[1] K. O. Friedrichs and D. H. Hyers, The existence of solitary waves, Comm. Pure Appl. Math. 7, 517-550 (1954) 